\title{
Neuroprotective Effect of 4T1 and Sertoli Cells Co- Transplantation in Animal Model of Brain Ischemia
}

\author{
Sara Milanizadeh ${ }^{1}$, Abbas Aliaghaei ${ }^{2}$, Mohammad Reza Bigdeli ${ }^{1,3^{*}}$ \\ ${ }^{1}$ Department of Animal Sciences and Biotechnology, Faculty of Life Sciences and Biotechnology, Shahid Beheshti University \\ Tehran, Iran \\ ${ }^{2}$ Department of Anatomy, Shahid Beheshti University of Medical Sciences, Tehran, Iran \\ ${ }^{3}$ Institute for Cognitive and Brain Sciences (ICBS), Shahid Beheshti University, Tehran, Iran
}

*Correspondence to Mohammad Reza Bigdeli, Tel: +98-2129902731, Fax: +98-2122441664 Email:

bigdelimohammadreza@yahoo.com

Received June 1, 2018 Accepted June 22, 2018 Published online September 30, 2018

\begin{abstract}
Introduction: Introducing neurotrophic factors is among several new approaches to enhance neural resistance to ischemic condition. Cancer cells such as 4T1 (an invasive breast carcinoma cell line) are the most potent cells with high viability in transplanted area. The 4T1 cells are derived from spontaneous tumors in BALB/C mouse with their pathogenicity which is limited to this animal. Sertoli cells (SCs) can be a proper candidate for increasing the survival of transplanted cells. The SCs not only suppress the immune system, but also secret many growth factors which increase neural survival. The aim of this study was to evaluate the possible neuroprotective effect of $4 \mathrm{~T} 1$ transplantation on middle cerebral artery occlusion (MCAO) rat model alone and in combination with SCs co-transplantation.

Methods: Rats were categorized into 5 experimental groups as follows: control, sham, SCs, 4T1, and $4 \mathrm{~T} 1+\mathrm{SCs}$ treated groups. For transplanting cells into right striatum, stereotaxic surgery was performed. In addition, ischemic surgery was induced after three days. Twenty-four hours after the reperfusion, neurological severity score (NSS), infarct volume, brain edema, and bloodbrain barrier (BBB) permeability were assessed in different areas of the brain including cortex, striatum, and piriform cortex-amygdala (Pir-Amy).

Results: The SCs, 4T1, and SCs+4T1 cells co-transplantation ameliorated neurological deficits and reduced infarct volume, brain edema, and BBB permeability compared to the control group. Conclusion: Transplanting 4T1 cancer cell along with SCs as a source of neurotrophic factors enhanced neural survival providing a potential new approach in cell therapy.

Keywords: Sertoli cells, Middle cerebral artery occlusion, BBB, Brain edema, Cell transplantation
\end{abstract}

Please cite this article as follows: Milanizadeh S, Aliaghaei A, Bigdeli MR. Neuroprotective effect of $4 \mathrm{~T} 1$ and Sertoli cells co-transplantation in animal model of brain ischemia. Int J Basic Sci Med. 2018;3(3):133139. doi:10.15171/ ijbms.2018.24.

\begin{abstract}
Introduction
Brain ischemia and neurological dysfunction are common causes of disabilities in the world. Hence, it is essential to find efficient and new ways to ameliorate neurological deficits. Hypoxic condition within the brain tissue increases lactate and enhances acidosis causing overproduction of free radicals. Oxidative stress, membrane pumps dysfunction, and disturbed $\mathrm{Ca}^{+2}$ hemostasis lead to neural damages in the brain. Ischemic preconditioning is a method which enhances neural survival in the brain.

Previous studies have demonstrated that transplantation of different cells releasing neurotrophic factors can enhance neural resistance to brain ischemia.
\end{abstract}

Among different cell types, cancer cells have potent survival ability to secrete high levels of growth factors. The 4T1 cells are invasive breast carcinoma cells which are derived from spontaneous tumors in $\mathrm{BALB} / \mathrm{c}$ mouse. Studies have demonstrated that these cells secrete high levels of growth factors such as granulocyte colonystimulating factor (G-CSF), macrophage colony-stimulating factor (M-CSF), vascular endothelial growth factor (VEGF), and interleukin 6 (IL-6) which can also be neuroprotective., ${ }^{1,2}$ Studies on condition media of the $4 \mathrm{~T} 1$ cells have shown that they secret high level of G-CSF. ${ }^{2}$

Furthermore, it has been indicated that the above-mentioned factors have neuroprotective effects. The G-CSF receptor exists in different regions of the

(C) 2018 The Author(s); Published by Zabol University of Medical Sciences. This is an open-access article distributed under the terms of the Creative Commons Attribution License (http://creativecommons.org/licenses/by/4.0), which permits unrestricted use, distribution, and reproduction in any medium, provided the original work is properly cited. 
brain including cortex, hippocampus, and substantial nigra. $^{3}$ It has been proven that subcutaneous and intraperitoneal injection of G-CSF results in neural protection in substantial nigra via increasing B-cell lymphoma 2 (Bcl-2) and decreasing Bcl-2-associated $\mathrm{X}$ protein ( $\mathrm{Bax}$ ) leading to the reduction of neural apoptosis., ${ }^{4,5}$ In addition, expression of G-CSF and its receptor are increased shortly after ischemia induction suggesting a role for G-CSF in endogenous response to ischemic conditions. ${ }^{6}$

The VEGF is another factor which is secreted by $4 \mathrm{~T} 1$ cells. Many studies have indicated that administration of VEGF decreased infarct volume, increased neural survival, and finally enhanced neurogenesis in the subventricular zone (SVZ) and hippocampus. It has also increased angiogenesis in brain ischemia ameliorating behavioral deficit. ${ }^{7}$ Transplantation of VEGF overexpressing transgenic cells showed neuroprotective effects in brain ischemia. ${ }^{8}$ Moreover, pathologic effects of $4 \mathrm{~T} 1$ cells are limited to $\mathrm{BALB} / \mathrm{c}$ mice therefore their transplantation in rats' brain are safe.

The Sertoli cells (SCs) support the cells in the testis and are known as the 'nurse cells' because of their ability to protect and increase the survival of germ cells. Their tightjunctions make a physical barrier that is important in protecting germs cells from unwanted immune reactions. These cells also suppress immune system by secreting interleukin 2 (IL-2) inhibitor factor. ${ }^{9}$ Transforming alpha and beta (TGF- $\alpha$ \& TGF- $\beta$ ) growth factors, insulin-like growth factor (IGF-I), fibroblast growth factor (FGF$2)$, glial cell line-derived neurotrophic factor (GDNF), neurotrophin-3 (NT-3), basic fibroblast growth factor (bFGF), and platelet-derived growth factor (PDGF) are among other secretions which present neuroprotective effects by the SCs. ${ }^{10,11}$ The injection of SCs into the spinal cord improved motor neuron survival in animal models of amyotrophic lateral sclerosis via their trophic factors. ${ }^{12}$ It has been demonstrated that injection of SCs derived growth factors exerted neuroprotective effects. For example, TGF- $\beta 1$, one of the SCs secreted factors, protected the structure of blood-brain barrier (BBB) via reduction of matrix metallopeptidase (MMP-2/9) expression. ${ }^{13}$ Besides, GDNF administration reduced neural apoptosis and improved neural disorders in ischemia and Parkinson disease. ${ }^{14}$

Previous studies have indicated that co-transplantation of SCs and islet xenografts increased the survival of transplanted cells by suppressing the immune system. ${ }^{15}$ Co-culture of SCs with endothelial and neural stem cells can also enhance their proliferation and survival. ${ }^{16}$ Another study has revealed that co-culture of SCs and bone marrow mesenchymal stem cells provide an environment that enhances stem cells proliferation via epidermal growth factor (EGF) signaling pathway. ${ }^{17}$ It has been revealed that allograft of dopaminergic neurons with SCs improved the survival of grafted neurons through modulating the immune responses. ${ }^{18}$ Regarding beneficial effects of $4 \mathrm{~T} 1$ and SCs cells toward cellular protection in allografts, the researchers of the present study evaluated the possible neuroprotective effect of 4T1 and SCs on infarct volume of brain damage, brain edema, and damages of BBB in mouse models of brain ischemia.

\section{Materials and Methods}

Animals

Sixty male Wistar rats (230 to $290 \mathrm{~g}$ ) were purchased from Pasteur Institute (Karaj, Iran). Rats were randomly divided into 5 groups and during all the experiments, they had free access to water and standard food. All the rats were kept at the same animal facility with a 12:12 hours of a dark-light cycle at $25^{\circ} \mathrm{C}$.

\section{Experimental Protocol}

The experimental groups consisted of control, sham, and 3 treatment groups receiving either $4 \mathrm{~T} 1$, SCs, or $4 \mathrm{~T} 1+\mathrm{SCs}$ cells by the stereotaxic method. In the control group, middle cerebral artery occlusion (MCAO) surgery was performed on the rats. A surgery without the entrance of coated nylon suture was performed in sham group to mimic the stress of surgery. In the treatment groups, 4T1, SCs, and 4T1+SCs cells were injected into the right striatum three days prior to the MCAO surgery.

\section{Focal Cerebral Ischemia}

The animals were anesthetized with intraperitoneally injected chloral hydrate ( $400 \mathrm{mg} / \mathrm{kg}$ ). The MCAO surgery was done according to the previously described method by Longa et al. ${ }^{19}$ Briefly, 20-22 mm of 3-0 silicone coated nylon suture was guided to reach bifurcation of the carotid throughout the internal carotid artery (ICA) until a mild resistance was recognized which meant the tip was stuck to the anterior cerebral artery and blocked the middle cerebral artery.

\section{Sertoli Cells Extraction}

Testes were removed from adult rats, cut into small pieces, and moved into the tubes containing media and antibiotics. Tissues were incubated at $37^{\circ} \mathrm{C}$ with trypsin $(0.25 \%)$ for 15 minutes. After aspiration of the trypsin, $1 \%$ collagenase solution was added and incubated for 15 minutes at $37^{\circ} \mathrm{C}$. Fetal bovine serum (FBS) was added and the samples were centrifuged. Cells were moved to the culture flasks with DMEM/F12 with 10\% FBS and vancomycin (25 mg/1 L DMEM). The SCs were incubated with Hoechst prior to the injection. The cells were then centrifuged and were counted with trypan blue. For SCs and $4 \mathrm{~T} 1+\mathrm{SCs}$ receiving groups, $5 \times 10^{5}$ and $2.5 \times 10^{5}$ (total of $\left.5 \times 10^{5}\right)$ cells were prepared for injection, respectively. ${ }^{20}$

\section{T1 Cells Culture}

The 4T1 cell line was obtained from Pasteur Institute (Tehran, Iran). Cells were cultured in DMEM/F12 with 
$10 \%$ FBS and vancomycin (25 mg/1 L DMEM). They were also counted before the injection as described before. The number of $5 \times 10^{5}$ and $2.5 \times 10^{5}$ cells were prepared for $4 \mathrm{~T} 1$ and $4 \mathrm{~T} 1+\mathrm{SCs}\left(\right.$ total of $5 \times 10^{5}$ ) receiving group, respectively.

\section{Cell Injection}

The animals were anesthetized and fixed in stereotaxic apparatus. According to the Paxinos atlas, cells were transplanted into the right striatum and the coordinates were as follows: Bregma $=0.96$, Lateral $=3 \mathrm{~mm}$, and DV $=5 \mathrm{~mm}$.

\section{Neurobehavioral Evaluation}

Twenty-four hours after reperfusion, NSS was measured according to the previously described method, ${ }^{21}$ which contained 5 parts (i.e., raise tail, sensory function, motor function, beam, and reflex tests) and the maximum score of 18 .

\section{Assessment of Infarct Volume}

The assessment was conducted by previously described method. ${ }^{22}$ Briefly, the rats were sacrificed 24 hours after reperfusion and their brains were kept in saline at $4^{\circ} \mathrm{C}$ for 10 minutes. The brains were then cut, with a thickness of $2 \mathrm{~mm}$, followed by incubation for 15 minutes with 2,3,5-Triphenyltetrazolium chloride $2 \%$ (TTC) at $37^{\circ} \mathrm{C}$. The following formula was used in this regard:

Corrected infarct volume $=$ Left hemisphere volume (Right hemisphere volume - Infarct volume)

\section{Analysis of Brain Water Content}

Wet weight (WW) and dry weight (DW) of different parts of the brain including cortex, striatum, and Pir-Amy were measured after 24 hours of incubation at $120^{\circ} \mathrm{C}$. At the end, brain water content was assessed by previously explained formula ${ }^{23}$ as follows:

Water content $=[($ Wet Weight - Dry Weight $) /$ Wet Weight $]^{*} 100$

\section{Assessment of BBB Permeability}

The BBB permeability was checked using Evans blue (Eb). In summary, 30 minutes after MCAO surgery, 2\%
EB $4 \mathrm{~mL} / \mathrm{kg}$ was injected into the tail vein. Perfusion with saline was performed and different parts of the brain were weighted 24 hours after the surgery (FEW,FH-300H, Japan). Brains were homogenized (Sonoplus Ultrasonic, HD2070-HD22500) in phosphate-buffered saline and were later mixed with trichloroacetic acid (60\%) and kept at $4^{\circ} \mathrm{C}$ for 30 minutes. Samples were centrifuged and the absorbance of supernatants were read at $610 \mathrm{~nm}$ by spectrophotometer (Analytikjena, Spercord, 210).The level of EB was stated as $\mu \mathrm{g} / \mathrm{g}$ of the brain tissue. ${ }^{23}$

\section{Statistical Analysis}

One-way analysis of variance (ANOVA) test was used to compare different parameters among the groups. As post hoc Tukey HSD test, LSD (the least significant difference) was employed and the statistical significance was represented as $P<0.05$. In addition, nonparametric Kruskal-Wallis test was applied for analyzing neurological severity scores (NSSs).

\section{Results}

Effect of 4T1 and SCs Allograft Transplantation and Cotransplantation on Neurologic Deficit Scores

All treatments including SCs, 4T1, and 4T1+SCs cotransplantation reduced total neurological NSS. The scores were 11 (range: 8-12), 9 (range: 5-11), and 9 (range: 5-13), respectively as compared to 14 (range: 13-15) in the control group (Table 1). The results revealed that sensory function and beam test were also improved significantly in all the treatment groups compared to the control group. Moreover, an improvement was noted in raise tail test in SCs and $4 \mathrm{~T} 1+\mathrm{SC}$ receiving groups compared to the control group. The distribution of NSS in different groups under investigation is provided in Table 1.

Effect of 4T1 and SCs Allograft Transplantation and Cotransplantation on Infarct Volume

Allograft transplantation of SCs, 4T1, and $4 \mathrm{~T} 1+\mathrm{SCs}$ co-transplantation three days before MCAO surgery decreased the total infarct volume respective to the control group.

The obtained results indicated a significant reduction

Table 1. Distribution of Neurological Severity Scores in Different Studied Groups

\begin{tabular}{|c|c|c|c|c|c|c|}
\hline \multirow{2}{*}{ Experimental Groups } & \multicolumn{3}{|c|}{ NSS in Each Group } & \multirow{2}{*}{ Total } & \multirow{2}{*}{ Median Score } & \multirow{2}{*}{$P$ Value } \\
\hline & $0-6$ & 6-12 & 16-18 & & & \\
\hline Control $(n=10)$ & 0 & 0 & 10 & 10 & 14 & - \\
\hline Sham $(n=10)$ & 8 & 2 & 0 & 10 & 4 & $<0.05$ \\
\hline $4 T 1 \quad(n=10)$ & 2 & 8 & 0 & 10 & 9 & $<0.05$ \\
\hline $4 \mathrm{~T} 1+\mathrm{SCs}(\mathrm{n}=10)$ & 1 & 8 & 1 & 10 & 9 & $<0.05$ \\
\hline SCs $(n=10)$ & 0 & 7 & 3 & 10 & 11 & $<0.05$ \\
\hline
\end{tabular}

Note 1. * NSS (neurological severity score) analysis of sham, SCs 4T1, and 4T1+SCs treated groups.

Note 2. Only the stress of MCAO surgery was repeated in the sham group and MCAO surgery was performed in the control group. Other groups received transplanted cell 3 days before the MCAO surgery. 
in infarct volume of the cortex and striatum in all the experimental groups. Infarct volume of Pir-Amy decreased significantly in SCs and 4T1+SCs treated groups while no notable change was detected in $4 \mathrm{~T} 1$ receiving group (Figure 1).

Effect of 4T1 and SCs Allograft Transplantation and Cotransplantation Transplant on Brain Water Content

Transplantation of SCs cells three days prior to MCAO surgery decreased brain edema in right cortex, Pir-Amy, and striatum regions in comparison with the control group. Brain edema of the right cortex and striatum in $4 \mathrm{~T} 1+\mathrm{SCs}$ treated group was reduced significantly while edema of Pir-Amy was not changed. No significant reduction was detected in $4 \mathrm{~T} 1$ treated group compared to the control group (Figure 2).

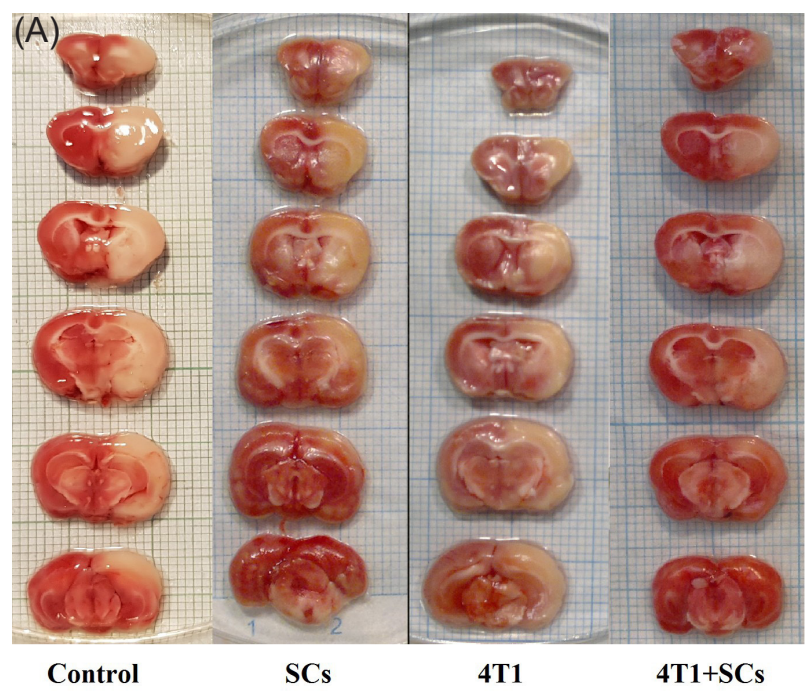

(B)

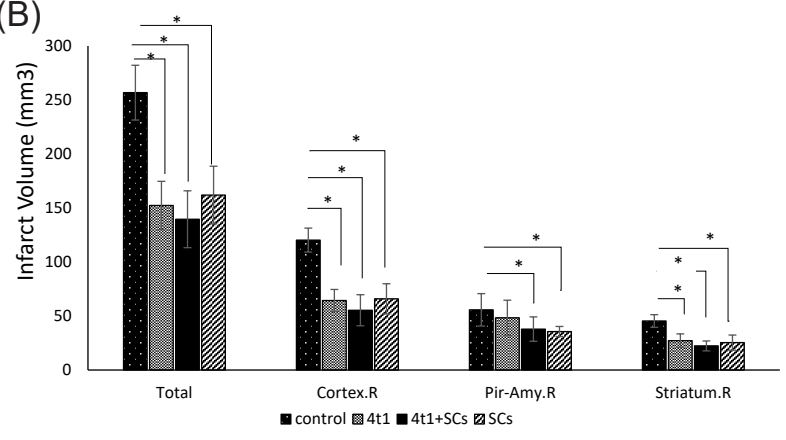

Figure 1. Infarct Volumes After Injection of SCs, 4T1, and 4T1+SCs Before MCAO Surgery. A: Coronal section of cell treated groups and control group are shown in each column with damaged areas in white. The 2,3,5-Triphenyltetrazolium chloride (TTC) staining revealed that SCs, 4T1, and 4T1 +SCs had a neuroprotective effect. B: Transplantation of SCs, $4 \mathrm{~T} 1$, and $4 \mathrm{~T} 1+\mathrm{SCs}$ decreased the total infarct volume related to the control group. Infarct volume of cortex and striatum markedly decreased in all the experimental groups. Infarct volume of Pir-Amy of SCs and $4 \mathrm{~T} 1+\mathrm{SCs}$ groups decreased significantly while no reduction was observed in Pir-Amy of 4T1 treated group compared to the control group. Values were represented as mean \pm SEM $(n=5)$. Note. The significance values were as $* P<0.05, * * P<0.01$, and *** $P<0.001$ compared to the control group.
Effect of 4T1 and SCs Allograft Transplantation and Cotransplantation on BBB Permeability

As compared with the control group, BBB permeability was reduced in the right cortex as well as striatum of SCs and $4 \mathrm{~T} 1+\mathrm{SCs}$ treated groups and also right Pir-Amy of SCs treated group while no significant reduction was found in other experimental groups (Figure 3).

\section{Discussion}

According to the results, it was noted that transplantation and co-transplantation of SCs and 4T1 cells resulted in improvement of neurological deficits after brain ischemia. The cell treatment also ameliorated the infarct volume in majority of the brain areas including cortex, Pir-Amy, and striatum. Moreover, BBB permeability and brain edema were improved in groups treated with SCs, but not in 4T1 receiving group probably because of the VEGF secretion

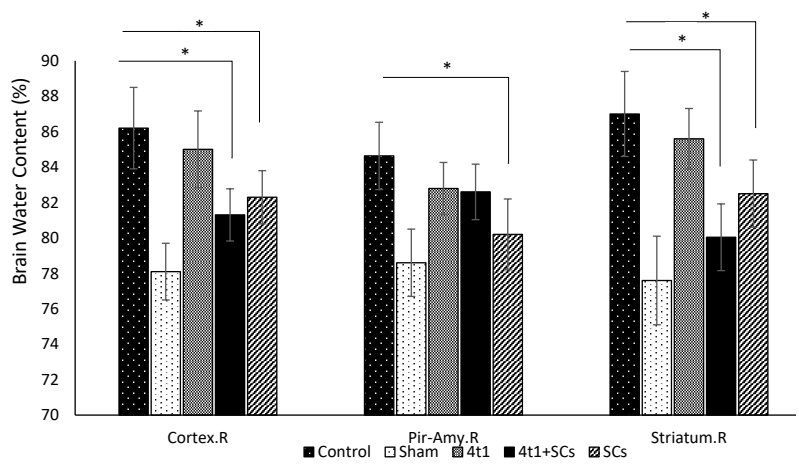

Figure 2. Effect of SCs, 4T1, 4T1+SCs Injection on Brain Edema in Right Cortex, Pir-Amy, and Striatum. In comparison with control group, a significant decrease was observed in the brain edema in the right cortex and striatum of SCs and 4T1+SCs treated groups. Besides, brain edema in Pir-Amy of SCs treated group significantly decreased as compared to the control group. Values were represented as mean $\pm \operatorname{SEM}(n=5)$. Note. The value of $* P<0.05$ was related to the control group.

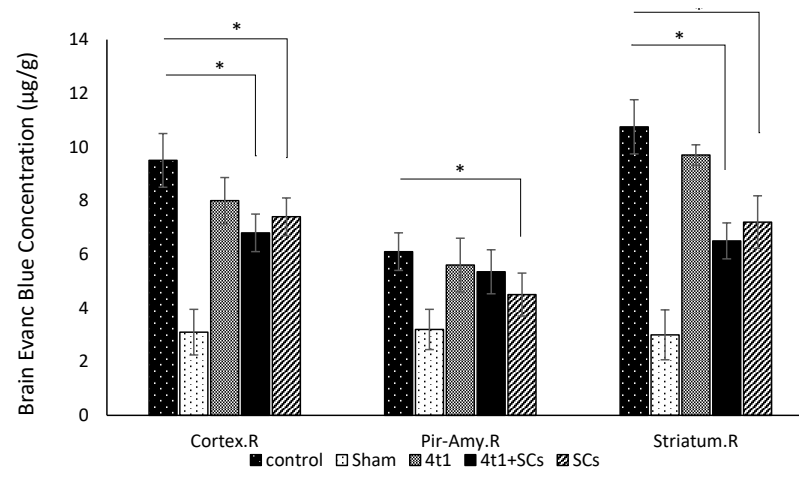

Figure 3. The Effects of SCs, 4T1, and $4 \mathrm{~T} 1+\mathrm{SCs}$ Transplantation on BBB by EB Concentration Analysis. The permeability of BBB decreased in the right cortex and striatum of both $\mathrm{SCs}$ and $4 \mathrm{~T} 1+\mathrm{SCs}$ treated groups as compared to the control. However, BBB permeability of right Pir-Amy decreased significantly only in SCs treated group. In addition, reduction of BBB permeability was also observed in 4T1 treated group, but it was not significant compared to control group. Values were shown as mean ${ }_{ \pm}$SEM $(\mathrm{n}=5)$. Note. ${ }^{*} P<0.05$ in comparison with control. 
by $4 \mathrm{~T} 1$ cells.

It has been revealed that intravenous injection of VEGF one hour after the ischemia increased BBB leakage and edema due to an elevation in brain microvascular permeability to blood plasma proteins. ${ }^{24}$ Studies have illustrated that VEGF had different effects on brain ischemia depending on the time of administration. ${ }^{25}$ In another study, it was also found that intravenous injection of VEGF one hour after ischemia failed to protect damages while its administration four hours after ischemia improved the neurological disorders. ${ }^{25}$ As it has been reported in another study, hypoxic preconditioning increased VEGF levels in an early stage of brain ischemia which resulted in dysregulation of $\mathrm{BBB} .{ }^{26}$ Although it seems that the administration of VEGF in early stage of brain ischemia may induce brain edema and BB disruption, many studies have revealed its neuroprotective roles in brain ischemia. It has been reported that VEGF reduced the infarct volume, ${ }^{27}$ improved neurological disorders, and increased the mesencephalic neurons survival. ${ }^{28}$ Studies have also indicated that VEGF had a direct mitogenic effect on neurons. ${ }^{29}$ The injection of VEGF 1 to 48 hours after the ischemia improved behavioral dysfunctions in rotarod and adhesion removal test. ${ }^{24}$ The VEGF enhanced neural survival and decreased toxicity in in vitro models of the brain ischemia. Researches have shown that transplantation of VEGF and BDNF secreting cells improved neural disorders. ${ }^{8}$ Transplantation of VEGFoverexpressed transgenic neural stem cells ameliorated behavioral dysfunction following the brain ischemia. ${ }^{30}$

Another main characteristic of 4T1 cell was their ability to produce G-CSF. ${ }^{1}$ Studies have shown that G-CSF reduced neural apoptosis in brain ischemia by reduction of caspase 3 through phosphoinositide 3-kinase/protein kinase (PI3/Akt) signaling pathway. ${ }^{3}$ They have also illustrated that injection of G-CSF decreased infarct volume, inflammation, and brain edema following brain ischemia via STAT (signal transducer and activator of transcription protein) signaling pathway. ${ }^{31}$ Similarly, it has been reported that the administration of human recombinant G-CSF ameliorated neurological disorders and decreased infarct volume by increasing $\mathrm{Bcl}-2$ expression. ${ }^{5}$ Besides, it has been indicated that G-CSF maintained $\mathrm{BBB}$ structure and permeability in ischemic conditions by increasing claudin-5 and decreasing intercellular adhesion molecule 1 (ICAM-I) and vascular cell adhesion protein (VCAM-I) in endothelial cells via PI3/Akt signaling pathway. ${ }^{32}$ In addition, it was found that G-CSF decreased hemisphere atrophy and infarct volume by increasing the vessel density and angiogenesis. ${ }^{33}$ According to the results obtained by another study, subcutaneous injection of G-CSF also reduced infarct volume and permeability of BBB following the ischemia. ${ }^{34}$

Moreover, transplantation of SCs in the striatum of Huntington animal models ameliorated locomotor abnormalities. ${ }^{12}$ Injection of microencapsulated SCs in the striatum, improved the neural deficits in the rat models of Huntington via producing growth factors. ${ }^{35}$ According to previous studies, cells with the ability of GDNF secretion can be valuable targets in cell therapy. The GDNF was found to decrease the neural apoptosis through increasing $\mathrm{Bcl}-2$ expression in Parkinson disease. ${ }^{14}$ Immunocytochemistry test results confirmed the identity of SCs and the researchers of the current study have revealed that these cells were able to secrete VEGF and GDNF (data is not represented). The GDNF decreased apoptosis and helped endothelial cells to keep their structure in BBB. It has also been demonstrated that GDNF reduced infarct volume and brain edema. ${ }^{36}$

Although SCs transplantation resulted in neural survival, SCs co-transplantation with other cells like 4T1 cells prolonged the survival of grafted cells. Infusion of SCs with grafted islet cells has been found to reduce peripheral lymphocyte activation and inflammatory cytokine levels. ${ }^{37}$ Moreover, ventral mesencephalon neutrons and SCs co-transplantation in striatum increased survival of grafted cells. ${ }^{38}$

\section{Conclusion}

Generally speaking, transplantation of $4 \mathrm{~T} 1$ cells resulted in neuroprotection probably through production of high level growth factors such as VEGF and G-CSF. The SCs co-transplantation with $4 \mathrm{~T} 1$ cells not only protected grafted cells system, but also enhanced neural survival.

\section{Ethical Approval}

The present study was based on the ethics standards for experiments involving animals issued by the Ethics Committee of Shahid Beheshti University, Tehran, Iran (ethical No. 396-2-020).

\section{Competing Interest}

Authors declare that they have no competing interests.

\section{Acknowledgment}

This work was supported by the Research Council of Shahid Beheshti University, Tehran, Iran.

\section{References}

1. Ravindranathan S, Smith SG, Nguyen K, Zaharoff DA. Colony stimulating factors secreted by irradiated autologous tumor cell vaccines inhibit immunity. J Immunother Cancer. 2015;3(Suppl 2):P448. doi:10.1186/2051-1426-3-s2-p448

2. Waight JD, Hu Q, Miller A, Liu S, Abrams SI. Tumor-derived G-CSF facilitates neoplastic growth through a granulocytic myeloid-derived suppressor cell-dependent mechanism. PLoS One. 2011;6(11):e27690. doi:10.1371/journal.pone.0027690

3. Schneider A, Kruger C, Steigleder T, et al. The hematopoietic factor G-CSF is a neuronal ligand that counteracts programmed cell death and drives neurogenesis. J Clin Invest. 2005;115(8):2083-2098. doi:10.1172/jci23559 
4. Meuer K, Pitzer C, Teismann P, et al. Granulocyte-colony stimulating factor is neuroprotective in a model of Parkinson's disease. J Neurochem. 2006;97(3):675-686. doi:10.1111/ j.1471-4159.2006.03727.x

5. Cao XQ, Arai H, Ren YR, et al. Recombinant human granulocyte colony-stimulating factor protects against MPTPinduced dopaminergic cell death in mice by altering $\mathrm{Bcl}-2 /$ Bax expression levels. J Neurochem. 2006;99(3):861-867. doi:10.1111/j.1471-4159.2006.04125.x

6. Kleinschnitz C, Schroeter M, Jander S, Stoll G. Induction of granulocyte colony-stimulating factor mRNA by focal cerebral ischemia and cortical spreading depression. Brain Res Mol Brain Res. 2004;131(1-2):73-78. doi:10.1016/j. molbrainres.2004.08.011

7. Sun Y, Jin K, Xie L, et al. VEGF-induced neuroprotection, neurogenesis, and angiogenesis after focal cerebral ischemia. J Clin Invest. 2003;111(12):1843-1851. doi:10.1172/jci17977

8. Zhou L, Lin Q, Wang P, et al. Enhanced neuroprotective efficacy of bone marrow mesenchymal stem cells cooverexpressing BDNF and VEGF in a rat model of cardiac arrest-induced global cerebral ischemia. Cell Death Dis. 2017;8(5):e2774. doi:10.1038/cddis.2017.184

9. Willing AE, Cameron DF, Sanberg PR. Sertoli cell transplants: their use in the treatment of neurodegenerative disease. Mol Med Today. 1998;4(11):471-477. doi:10.1016/s13574310(98)01355-0

10. Skinner MK. Sertoli cell secreted regulatory factors. Sertoli cell biology; 2005:107-120. doi:10.1016/B978-012647751$1 / 50009-\mathrm{X}$

11. Chen SR, Tang JX, Cheng JM, et al. Loss of Gata4 in Sertoli cells impairs the spermatogonial stem cell niche and causes germ cell exhaustion by attenuating chemokine signaling. Oncotarget. 2015;6(35):37012-37027. doi:10.18632/ oncotarget.6115

12. Hemendinger R, Wang J, Malik S, et al. Sertoli cells improve survival of motor neurons in SOD1 transgenic mice, a model of amyotrophic lateral sclerosis. Exp Neurol. 2005;196(2):235243. doi:10.1016/j.expneurol.2005.07.025

13. Cai Y, Liu X, Chen W, et al. TGF-betal prevents bloodbrain barrier damage and hemorrhagic transformation after thrombolysis in rats. Exp Neurol. 2015;266:120-126. doi:10.1016/j.expneurol.2015.02.013

14. d'Anglemont de Tassigny X, Pascual A, Lopez-Barneo J. GDNF-based therapies, GDNF-producing interneurons, and trophic support of the dopaminergic nigrostriatal pathway. Implications for Parkinson's disease. Front Neuroanat. 2015;9:10. doi:10.3389/fnana.2015.00010

15. Dufour JM, Rajotte RV, Kin T, Korbutt GS. Immunoprotection of rat islet xenografts by cotransplantation with sertoli cells and a single injection of antilymphocyte serum. Transplantation. 2003;75(9):1594-1596. doi:10.1097/01. tp.0000058748.00707.88

16. Fan $\mathrm{P}, \mathrm{He} \mathrm{L}, \mathrm{Pu} \mathrm{D}$, et al. Testicular Sertoli cells influence the proliferation and immunogenicity of co-cultured endothelial cells. Biochem Biophys Res Commun. 2011;404(3):829-833. doi:10.1016/j.bbrc.2010.12.068

17. Tian H, Guo M, Zhuang Y, Chu J, Zhang S. Enhanced proliferation of bone marrow mesenchymal stem cells by co-culture with TM4 mouse Sertoli cells: involvement of the EGF/PI3K/AKT pathway. Mol Cell Biochem. 2014;393(1-
2):155-164. doi:10.1007/s11010-014-2055-x

18. Cheng CY, Jhao YT, Chou TK, et al. Evaluating the allograft effects of Sertoli cells and embryonic VM tissues in a Parkinsonian rat model using [18F] FE-PE2I/animal-PET. J Nucl Med. 2017;58(suppl 1):271.

19. Longa EZ, Weinstein PR, Carlson S, Cummins R. Reversible middle cerebral artery occlusion without craniectomy in rats. Stroke. 1989;20(1):84-91. doi:10.1161/01.STR.20.1.84

20. Milanizadeh S, Zuwarali KNN, Aliaghaei A, Bigdeli MR. Therapeutic Potential of Pretreatment with Allograft Sertoli Cells Transplantation in Brain Ischemia by Improving Oxidative Defenses. J Mol Neurosci. 2018;64(4):533-542. doi:10.1007/s12031-018-1054-x

21. Long J, Cai L, Li J, Zhang L, Yang H, Wang T. JNK3 involvement in nerve cell apoptosis and neurofunctional recovery after traumatic brain injury. Neural Regen Res. 2013;8(16):14911499. doi:10.3969/j.issn.1673-5374.2013.16.006

22. Lin Y, Zhang JC, Fu J, et al. Hyperforin attenuates brain damage induced by transient middle cerebral artery occlusion (MCAO) in rats via inhibition of TRPC6 channels degradation. J Cereb Blood Flow Metab. 2013;33(2):253-262. doi:10.1038/ jcbfm.2012.164

23. Bigdeli MR, Hajizadeh $S$, Froozandeh $M$, Rasulian B, Heidarianpour A, Khoshbaten A. Prolonged and intermittent normobaric hyperoxia induce different degrees of ischemic tolerance in rat brain tissue. Brain Res. 2007;1152:228-233. doi:10.1016/j.brainres.2007.03.068

24. Zhang ZG, Zhang L, Jiang Q, et al. VEGF enhances angiogenesis and promotes blood-brain barrier leakage in the ischemic brain. J Clin Invest. 2000;106(7):829-838. doi:10.1172/jci9369

25. van Bruggen $\mathrm{N}$, Thibodeaux $\mathrm{H}$, Palmer JT, et al. VEGF antagonism reduces edema formation and tissue damage after ischemia/reperfusion injury in the mouse brain. J Clin Invest. 1999;104(11):1613-1620. doi:10.1172/jci8218

26. Chi OZ, Mellender SJ, Barsoum S, Liu X, Weiss HR. Hypoxic Preconditioning Increases Blood-Brain Barrier Disruption in the Early Stages of Cerebral Ischemia. Curr Neurovasc Res. 2017;14(1):26-31. doi:10.2174/1567202614666161104114821

27. Hayashi T, Abe K, Itoyama Y. Reduction of ischemic damage by application of vascular endothelial growth factor in rat brain after transient ischemia. J Cereb Blood Flow Metab. 1998;18(8):887-895. doi:10.1097/00004647-199808000-00009

28. Silverman WF, Krum JM, Mani N, Rosenstein JM. Vascular, glial and neuronal effects of vascular endothelial growth factor in mesencephalic explant cultures. Neuroscience. 1999;90(4):1529-1541.

29. Jin K, Zhu Y, Sun Y, Mao XO, Xie L, Greenberg DA. Vascular endothelial growth factor (VEGF) stimulates neurogenesis in vitro and in vivo. Proc Natl Acad Sci U S A. 2002;99(18):1194611950. doi:10.1073/pnas.182296499

30. Zhu W, Mao Y, Zhao Y, et al. Transplantation of vascular endothelial growth factor-transfected neural stem cells into the rat brain provides neuroprotection after transient focal cerebral ischemia. Neurosurgery. 2005;57(2):325-333; discussion 325-333. doi:10.1227/01.NEU.0000166682.50272. $\mathrm{BC}$

31. Shuai K. Modulation of STAT signaling by STAT-interacting proteins. Oncogene. 2000;19(21):2638-2644. doi:10.1038/ sj.onc. 1203522

32. Li L, McBride DW, Doycheva D, et al. G-CSF attenuates 
neuroinflammation and stabilizes the blood-brain barrier via the PI3K/Akt/GSK-3beta signaling pathway following neonatal hypoxia-ischemia in rats. Exp Neurol. 2015;272:135144. doi:10.1016/j.expneurol.2014.12.020

33. Lee ST, Chu K, Jung $\mathrm{KH}$, et al. Granulocyte colonystimulating factor enhances angiogenesis after focal cerebral ischemia. Brain Res. 2005;1058(1-2):120-128. doi:10.1016/j. brainres.2005.07.076

34. Lu CZ, Xiao BG. G-CSF and neuroprotection: a therapeutic perspective in cerebral ischaemia. Biochem Soc Trans. 2006;34(Pt 6):1327-1333. doi:10.1042/bst0341327

35. Luca G, Bellezza I, Arato I, et al. Terapeutic Potential of Microencapsulated Sertoli Cells in Huntington Disease. CNS Neurosci Ther. 2016;22(8):686-690. doi:10.1111/cns.12569
36. Kobayashi T, Ahlenius H, Thored P, Kobayashi R, Kokaia Z, Lindvall $\mathrm{O}$. Intracerebral infusion of glial cell line-derived neurotrophic factor promotes striatal neurogenesis after stroke in adult rats. Stroke. 2006;37(9):2361-2367. doi:10.1161/01. STR.0000236025.44089.e1

37. Li Y, Xue WJ, Tian XH, et al. Study on systemic immune tolerance induction in rat islet transplantation by intravenous infusion of Sertoli cells. Transplantation. 2010;89(12):14301437. doi:10.1097/TP.0b013e3181da607e

38. Shamekh R, Newcomb J, Mallery J, et al. Survival of rat or mouse ventral mesencephalon neurons after cotransplantation with rat sertoli cells in the mouse striatum. Cell Transplant. 2005;14(8):551-564. doi:10.3727/000000005783982747 